PROCEEDINGS OF THE

AMERICAN MATHEMATICAL SOCIETY

Volume 130 , Number 11, Pages 3401-3408

S 0002-9939(02)06457-2

Article electronically published on April 22, 2002

\title{
PARABOLIC SUPER MEAN VALUE INEQUALITY
}

\author{
CHIUNG-JUE SUNG
}

(Communicated by Bennett Chow)

\begin{abstract}
In this note, we show that a type of mean value inequality for the positive supersolutions to the heat equation on a Riemannian manifold is equivalent to a version of local heat kernel lower bounds. We also show that the global Gaussian type heat kernel lower bound implies that the manifold has the Liouville property.
\end{abstract}

\section{$\S 0$. INTRODUCTION}

In the theory of elliptic and parabolic differential equations, the Harnack type inequalities have played an important role. It is remarkable that a type of parabolic Harnack inequality on a Riemannian manifold may be characterized in terms of the geometry of the manifold. Let us first recall several definitions.

Definition 0.1. The Riemannian manifold $M$ satisfies the parabolic Harnack inequality if for some constants $0<\epsilon<\eta<1$ and $0<\delta<1$, there exists a constant $c$ such that every nonnegative solution $u(x, t)$ to

$$
\left(\Delta-\frac{\partial}{\partial t}\right) u(x, t)=0
$$

on $B_{p}(r) \times\left[s-r^{2}, s\right]$ satisfies

$$
\sup _{\left[s-\delta r^{2}, s-\eta r^{2}\right] \times B_{p}(\delta r)} u \leq c \inf _{\left[s-\epsilon r^{2}, s\right) \times B_{p}(\delta r)} u .
$$

Definition 0.2. The Riemannian manifold $M$ is said to have the volume doubling property if there exists a constant $c$ such that $V_{x}(2 r) \leq c V_{x}(r)$ for all $x \in M$ and $r>0$, where $V_{x}(r)$ denotes the volume of the geodesic ball $B_{x}(r)$ centered at $x$ of radius $r$.

Definition 0.3. The Riemannian manifold $M$ is said to have the weak Poincaré inequality if for some constants $0<\delta<1$ and $C>0$,

$$
\inf _{c \in \mathbb{R}} \int_{B_{p}(\delta r)}(f-c)^{2} \leq C r^{2} \int_{B_{p}(r)}|\nabla f|^{2}
$$

for all $p \in M, r>0$ and $f \in C^{\infty}(M)$.

Received by the editors December 29, 2000 and, in revised form, June 15, 2001.

2000 Mathematics Subject Classification. Primary 58J35.

The author's research was partially supported by NSC grant.

(C)2002 American Mathematical Society 
The results of Grigor'yan [G] and Saloff-Coste [Sc] then imply that the Riemannian manifold $M$ satisfies the parabolic Harnack inequality if and only if it has both the volume doubling property and the weak Poincaré inequality. Based on this characterization, it was pointed out by Wang [W] that the validity of the heat kernel bounds is equivalent to the parabolic Harnack inequality on $M$,

$$
\frac{c_{1}}{V_{x}(\sqrt{t})} \exp \left(-\frac{r^{2}(x, y)}{c_{2} t}\right) \leq H(x, y, t) \leq \frac{c_{3}}{V_{x}(\sqrt{t})} \exp \left(-\frac{r^{2}(x, y)}{c_{4} t}\right),
$$

where $c_{1}, c_{2}, c_{3}$ and $c_{4}$ are constants.

The mean value type inequalities for both the subsolutions and the supersolutions to the elliptic or parabolic equations are also important. In a recent paper by $\mathrm{Li}$ and Wang $[\mathrm{L}-\mathrm{W}$, they studied a type of mean value inequalities for subsolutions and obtained some interesting results. We shall refer the reader to their paper for the details. Our purpose here is to consider the mean value inequality for the supersolutions.

Definition 0.4. The Riemannian manifold $M$ is said to have parabolic super mean value inequality if for some $0<\delta<1,0<\eta<\epsilon<1$, there exists a constant $c>0$ such that every nonnegative supersolution $u(x, t)$ to

$$
\left(\Delta-\frac{\partial}{\partial t}\right) u(x, t) \leq 0
$$

on $B_{p}(r) \times\left[s-r^{2}, s\right]$ satisfies

$$
u(p, s) \geq \frac{C}{V_{p}(\delta r) r^{2}} \int_{s-\epsilon r^{2}}^{s-\eta r^{2}} \int_{B_{p}(\delta r)} u(x, t) d x d t
$$

for any $p \in M$ and $r>0$.

Notice that this is a weaker version of the usual mean value inequality appearing in the literature as we only require the inequality to be valid at the center of the ball. We will show that the parabolic super mean value inequality is more or less equivalent to a type of lower bound for the Dirichlet heat kernel on the geodesic balls. In fact, we will show that it is also equivalent to the parabolic Harnack inequality under the volume doubling assumption. This will be shown in Section 1. In Section 2, we consider a type of Gaussian lower bound for the minimal heat kernel of a complete Riemannian manifold. We show that the manifold necessarily has both the volume doubling property and the Liouville property, namely, all the bounded harmonic functions must be constant. As a consequence, the connected sum of two nonparabolic manifolds cannot possibly admit such a Gaussian lower bound for its heat kernel.

\section{$\S 1$ Parabolic super mean value inequality}

We start by showing that the parabolic super mean value inequality implies a type of heat kernel lower bound. The proofs of the following two propositions are along classic lines and included for the convenience of the reader.

Proposition 1.1. Let $M$ be a complete manifold satisfying the parabolic super mean value inequality. Let $H(x, y, t)$ be the Dirichlet heat kernel of the ball $B_{p}(r)$. Then there exist constants $c_{1}$ and $c_{2}$ such that, for $x$ and $y$ in $B_{p}\left(\frac{\delta r}{8}\right)$,

$$
H\left(x, y, c_{1} r^{2}\right) \geq \frac{c_{2}}{V_{p}(r)} .
$$


Proof. Define the function

$$
u(x, t)= \begin{cases}\int_{B_{p}\left(\frac{\delta r}{4}\right)} H(x, z, t) d z & \text { if } t>0, \\ 1 & \text { if } t \leq 0 .\end{cases}
$$

Then $u(x, t) \geq 0$ and $\left(\delta-\frac{\partial}{\partial t}\right) u(x, t)=0$ on $B_{p}\left(\frac{\delta r}{4}\right) \times(-\infty, \infty)$. By the parabolic super mean value inequality, we have

$$
u(y, s) \geq \frac{c}{V_{y}(\delta R) R^{2}} \int_{s-\epsilon R^{2}}^{s-\eta R^{2}} \int_{B_{y}(\delta R)} u(x, t) d x d t
$$

if $B_{y}(\delta R) \subset B_{p}\left(\frac{\delta r}{4}\right)$. So $u(y, s) \geq c$ if $s \leq \eta R^{2}$ and $B_{y}(\delta R) \subset B_{p}(r)$. In particular, letting $R=\frac{r}{8}$, we get

$$
\int_{B_{p}\left(\frac{\delta r}{4}\right)} H(y, z, s) d z \geq c
$$

for $y \in B_{p}\left(\frac{\delta r}{8}\right)$ and $0<s \leq \frac{\eta}{64} r^{2}$. Now applying the parabolic super mean value inequality to the heat kernel $H(y, x, t)$, we obtain

$$
H(y, x, \tau) \geq \frac{c}{V_{x}\left(\delta r_{1}\right) r_{1}^{2}} \int_{\tau-\epsilon r_{1}^{2}}^{\tau-\eta r_{1}^{2}} \int_{B_{x}\left(\delta r_{1}\right)} H(y, z, s) d y d s
$$

if $B_{x}\left(\delta r_{1}\right) \subset B_{p}(r)$ and $\tau-\epsilon r_{1}^{2}>0$. For $x \in B_{p}\left(\frac{\delta r}{8}\right)$, choose $r_{1}=\frac{r}{2}$. Then $B_{x}\left(\delta r_{1}\right) \subset B_{p}(r)$. Also, $B_{x}\left(\delta r_{1}\right) \supset B_{p}\left(\frac{\delta r}{4}\right)$. Setting $\tau=\epsilon r_{1}^{2}+\frac{\eta}{2} R^{2}$ in (1.1) and using (1.2), we conclude

$$
\begin{aligned}
H\left(y, x, \epsilon r_{1}^{2}+\frac{\eta}{2} R^{2}\right) & \geq \frac{c}{V_{x}\left(\delta r_{1}\right) r_{1}^{2}} \int_{\frac{\eta}{2} R^{2}}^{\frac{\eta}{2} R^{2}+(\epsilon-\eta) r_{1}^{2}} \int_{B_{x}\left(\delta r_{1}\right)} H(y, z, s) d z d s \\
& \geq \frac{c}{V_{p}(r) r^{2}} \int_{\frac{\eta}{2} R^{2}}^{\frac{\eta}{2}(1+\epsilon-\eta) R^{2}} \int_{B_{p}\left(\frac{\delta r}{4}\right)} H(y, z, s) d z d s \\
& \geq \frac{c}{V_{p}(r) r^{2}} \frac{\eta(\epsilon-\eta)}{2} R^{2} \\
& =\frac{c}{V_{p}(r)}
\end{aligned}
$$

The proof is complete.

The following result says that a type of inverse holds for Proposition 1.1.

Proposition 1.2. If the Dirichlet heat kernel $H(x, y, t)$ of any geodesic ball $B_{p}(r)$ of $M$ satisfies

$$
H\left(x, y, c_{1} r^{2}\right) \geq \frac{c_{2}}{V_{p}\left(c_{3} r\right)}
$$

for $x$ and $y$ in $B_{p}(\delta r)$, where $c_{1}, c_{2}$ and $c_{3}$ are constants independent of $p$ and $r$, and $0<\delta<1$, then for $0<\eta<\epsilon<1$, a positive supersolution $u(x, t)$ to

$$
\left(\Delta-\frac{\partial}{\partial t}\right) u(x, t) \leq 0
$$

on $B_{q}(R) \times\left[s-R^{2}, s\right]$ satisfies

$$
u(q, s) \geq \frac{c}{V_{q}\left(c_{3} R\right) R^{2}} \int_{s-\epsilon R^{2}}^{s-\eta R^{2}} \int_{B_{q}(\delta \sqrt{\eta} R)} u(x, t) d x d t .
$$


Proof. Let $H(x, y, t)$ be the Dirichlet heat kernel of the ball $B_{q}(R)$. By the maximum principle, since $u(x, t)$ is a positive supersolution, we get

$$
u(q, s) \geq \int_{B_{q}(R)} H(q, y, s-\tau) u(y, \tau) d y
$$

for $s-\tau>0$. We now claim that for $r \leq R$,

$$
H\left(x, y, c_{1} r^{2}\right) \geq \frac{c_{2}}{V_{q}\left(c_{3} r\right)}
$$

for $x$ and $y$ in $B_{q}(\delta r)$. In fact, let $K(x, y, t)$ be the Dirichlet heat kernel of the ball $B_{q}(r)$. Then we have $K(x, y, t) \leq H(x, y, t)$ for $x$ and $y$ in $B_{q}(r)$, and all $t \geq 0$. Using the assumption, we have

$$
K\left(x, y, c_{1} r^{2}\right) \geq \frac{c_{2}}{V_{q}\left(c_{3} r\right)}
$$

n for $x$ and $y$ in $B_{q}(\delta r)$. So the claim follows.

Applying (1.4) to (1.3), we have

$$
u(q, s) \geq \frac{c_{2}}{V_{q}\left(c_{3} \sqrt{(s-\tau) / c_{1}}\right)} \int_{B_{q}\left(\delta \sqrt{(s-\tau) / c_{1}}\right)} u(y, \tau) d y,
$$

where $0<s-\tau \leq c_{1} R^{2}$. Integrating (1.5) with respect to $\tau$, we conclude that

$$
\begin{aligned}
u(q, s) & =\frac{1}{(\epsilon-\eta) R^{2}} \int_{s-\epsilon R^{2}}^{s-\eta R^{2}} u(q, s) d \tau \\
& \geq \frac{1}{(\epsilon-\eta) R^{2}} \int_{s-\epsilon R^{2}}^{s-\eta R^{2}} \frac{c_{2}}{V_{q}\left(c_{3} \sqrt{(s-\tau) / c_{1}}\right)} \int_{B_{q}\left(\delta \sqrt{(s-\tau) / c_{1}}\right)} u(y, \tau) d y d \tau \\
& \geq \frac{c}{V_{q}\left(c_{3} R\right) R^{2}} \int_{s-\epsilon R^{2}}^{s-\eta R^{2}} \int_{B_{q}\left(\delta \sqrt{\eta / c_{1}} R\right)} u(y, \tau) d y d \tau .
\end{aligned}
$$

The proof is complete.

The following lemma is due to Kusuoka and Stroock $[\mathrm{K}-\mathrm{S}]$; see also $[\mathrm{Sc}]$.

Lemma 1.3. Let $M$ be a complete manifold. Suppose that the Neumann heat kernel $H(x, y, t)$ of the geodesic ball $B_{p}(r)$ satisfies

$$
H\left(x, y, c_{1} r^{2}\right) \geq \frac{c_{2}}{V_{p}\left(c_{3} r\right)}
$$

for $x$ and $y$ in $B_{p}(\delta r)$, where $0<\delta<1$ and $c_{1}, c_{2}$ and $c_{3}$ are some constants. Then for $f \in C^{\infty}(M)$,

$$
\inf _{c \in \mathbb{R}} \int_{B_{p}(\delta r)}(f-c)^{2} \leq \frac{2 c_{1} V_{p}\left(c_{3} r\right) r^{2}}{c_{2} V_{p}(\delta r)} \int_{B_{p}(r)}|\nabla f|^{2} .
$$

Note that the Neumann heat kernel is always bounded from below by the Dirichlet heat kernel for any geodesic ball by the maximum principle. Combining the results of Proposition 1.1, Proposition 1.2 and Lemma 1.3, and using the result of Grigor'yan [G] and Saloff-Coste [Sc], we obtain the following theorem.

Theorem 1.4. For a complete manifold $M$ with volume doubling property, the following are equivalent:

1. The parabolic super mean value inequality holds on $M$.

2. The parabolic Harnack inequality holds on $M$. 
3. The Dirichlet heat kernel $H(x, y, t)$ of any geodesic ball $B_{p}(r)$ of $M$ satisfies

$$
H\left(x, y, c_{1} r^{2}\right) \geq \frac{c_{2}}{V_{p}\left(c_{3} r\right)}
$$

for $x$ and $y$ in $B_{p}(\delta r)$, where $c_{1}, c_{2}$ and $c_{3}$ are constants independent of $p$ and $r$, and $0<\delta<1$.

We remark that a local version of the theorem is also true by the same proof.

\section{§2. Heat kernel LOWER Bound and the Liouville PRoperty}

In this section, we will derive some necessary conditions for a complete manifold to admit a type of global Gaussian lower bound for its minimal heat kernel. We will show, in particular, that the manifold has to satisfy the volume doubling property and the Liouville property, namely, all the bounded harmonic functions must be constant. Let us start with the following lemma.

Lemma 2.1. Let $M$ be a complete Riemannian manifold. Suppose that its minimal heat kernel $H(x, y, t)$ satisfies the lower bound

$$
H(x, y, t) \geq \frac{c_{1}}{V_{x}\left(c_{2} \sqrt{t}\right)} \exp \left(-\frac{r^{2}(x, y)}{c_{3} t}\right),
$$

where $c_{1}, c_{2}$ and $c_{3}$ are constants. Then $M$ has the volume doubling property.

Proof. Note that by the minimality of the heat kernel $H(x, y, t)$, we have

$$
\int_{M} H(x, y, t) d y \leq 1
$$

Using the lower bound of $H(x, y, t)$, we get

$$
\begin{aligned}
1 & \geq \int_{B_{x}\left(2 c_{2} \sqrt{t}\right)} H(x, y, t) d y \\
& \geq \frac{c_{1} e^{-4 c_{2}^{2} / c_{3}} V_{x}\left(2 c_{2} \sqrt{t}\right)}{V_{x}\left(c_{2} \sqrt{t}\right)} .
\end{aligned}
$$

By (2.1), we conclude that

$$
V_{x}\left(2 c_{2} \sqrt{t}\right) \leq c V_{x}\left(c_{2} \sqrt{t}\right)
$$

Since both $x \in M$ and $t>0$ are arbitrary, one sees that $M$ has the volume doubling property. The proof is complete.

Utilizing the previous lemma, we now show $M$ has the Liouville property.

Proposition 2.2. Let $M$ be a complete manifold with its minimal heat kernel satisfying the lower bound as in Lemma 2.1. Then the Liouville property holds on M. That is, every bounded harmonic function on $M$ must be constant.

Proof. Let $u(x)$ be a bounded harmonic function on $M$. Consider the function $v=\sup _{M} u-u$. Since $v$ is bounded and $\delta v=0$, we have

$$
v(x)=\int_{M} H(x, y, t) v(y) d y .
$$

In fact, as $M$ has the volume doubling property, it has polynomial volume growth and is stochastically complete. In particular, the bounded solution to the heat 
equation is uniquely determined by its initial data. Since $\inf _{M} v=0$, for any $\epsilon>0$ there exists $x_{\epsilon}$ such that $v\left(x_{\epsilon}\right)<\epsilon$. So

$$
\int_{M} H\left(x_{\epsilon}, y, t\right) v(y) d y<\epsilon .
$$

Using the lower bound of the heat kernel, we obtain from (2.2) that

We rewite it as

$$
\frac{1}{V_{x_{\epsilon}}\left(c_{2} \sqrt{t}\right)} \int_{B_{x_{\epsilon}}\left(c_{2} \sqrt{t}\right)} v(y) d y<c \epsilon .
$$

$$
\frac{1}{V_{x_{\epsilon}}\left(c_{2} \sqrt{t}\right)} \int_{B_{x_{\epsilon}}\left(c_{2} \sqrt{t}\right)} u(y) d y \geq \sup _{M} u-c \epsilon .
$$

Similarly, if we consider $w=u-\inf _{M} u$, then $\inf _{M} w=0$ and there exist $\tilde{x_{\epsilon}}$ such that

$$
\epsilon>\int_{M} H\left(\tilde{x_{\epsilon}}, y, t\right) v(y) d y \geq \frac{C}{V_{\tilde{x_{\epsilon}}}\left(c_{2} \sqrt{t}\right)} \int_{B_{\tilde{x_{\epsilon}}}\left(c_{2} \sqrt{t}\right)} v(y) d y,
$$

which can be written as

$$
C \epsilon+\inf _{M} u \geq \frac{1}{V_{\tilde{x_{\epsilon}}}\left(c_{2} \sqrt{t}\right)} \int_{B_{\tilde{x_{\epsilon}}}\left(c_{2} \sqrt{t}\right)} u(y) d y .
$$

Combining (2.3) and (2.4), we get

$$
\begin{aligned}
\sup _{M} u-c \epsilon & \leq \frac{1}{V_{x_{\epsilon}}\left(c_{2} \sqrt{t}\right)} \int_{B_{\tilde{x_{\epsilon}}}\left(c_{2} \sqrt{t}+d\left(x_{\epsilon}, \tilde{x_{\epsilon}}\right)\right)} u(y) d y \\
& \leq \frac{V_{\tilde{x_{\epsilon}}}\left(c_{2} \sqrt{t}+d\left(x_{\epsilon}, \tilde{x_{\epsilon}}\right)\right)}{V_{x_{\epsilon}}\left(c_{2} \sqrt{t}\right)}\left(C \epsilon+\inf _{M} u\right) .
\end{aligned}
$$

We now claim that for any $p \in M$ and $r_{0}>0$,

$$
\liminf _{r \rightarrow \infty} \frac{V_{p}\left(r+r_{0}\right)}{V_{p}(r)}=1 .
$$

Otherwise, there exists $R \geq r_{0}$ such that for $r \geq R$,

$$
\frac{V_{p}\left(r+r_{0}\right)}{V_{p}(r)} \geq C>1 \text {. }
$$

So

$$
\frac{V_{p}(r+R)}{V_{p}(r)} \geq C
$$

Iterating the inequality $k$ times, we have

$$
V_{p}(k R) \geq C^{k-1} V_{p}(R) .
$$

This implies that $V_{p}(r)$ is of exponential growth as $C>1$. But $M$ has the volume doubling property by Lemma 2.1 and $V_{p}(r)$ must be of polynomial growth. The contradiction proves our claim. Letting $t \rightarrow \infty$ and noting the claim, we conclude from (2.5)

$$
C \epsilon+\inf _{M} u \geq \sup _{M} u-c \epsilon .
$$

Since $\epsilon>0$ is arbitrary, we have $\inf _{M} u=\sup _{M} u$ and $u$ is constant. The proof is complete. 
Since the connected sum of two nonparabolic manifolds admits nonconstant bounded harmonic functions (see [S-T-W]), this implies that the heat kernel on such manifolds cannot possibly admit a Gaussian type lower bound as in Lemma 2.1. Finally, we follow $[\mathrm{L}$ to give a proof of the following result by using Lemma 2.1 and Proposition 2.2.

Theorem 2.3. Let $M$ be a complete manifold with its minimal heat kernel satisfying a lower bound as in Lemma 2.1. Then for any bounded superharmonic function $f$ on $M$,

$$
\inf _{M} f=\lim _{r \rightarrow \infty} \frac{1}{V_{p}(r)} \int_{B_{p}(r)} f(x) d x .
$$

Proof. We may assume without loss of the generality that $f$ satisfies $\inf _{M} f=0$. Let $u(x, t)$ be the solution of the heat equation with $f$ as the initial data. Then, by $[\mathrm{L}$, we know $u(x, t)$ is a decreasing function of $t$. Hence it must converge uniformly on compact subsets of $M$ to a bounded harmonic function as $t \rightarrow \infty$. Since $M$ has the Liouville property by Proposition 2.2, the limit function must be constant and the constant must be zero as inf $f=0$. On the other hand,

$$
\begin{aligned}
u(p, t) & =\int_{M} H(p, y, t) f(y) d y \\
& \geq \frac{c_{1}}{V_{p}\left(c_{2} \sqrt{t}\right)} \int_{B_{p}\left(c_{2} \sqrt{t}\right)} \exp \left(-\frac{r^{2}(p, y)}{c_{3} t}\right) f(y) d y \\
& \geq \frac{c}{V_{p}\left(c_{2} \sqrt{t}\right)} \int_{B_{p}\left(c_{2} \sqrt{t}\right)} f(y) d y .
\end{aligned}
$$

Thus,

$$
\lim _{t \rightarrow \infty} \frac{1}{V_{p}\left(c_{2} \sqrt{t}\right)} \int_{B_{p}\left(c_{2} \sqrt{t}\right)} f(y) d y=0
$$

and the theorem is proved.

\section{ACKNOWLEDGMENTS}

The author would like to thank Professors Peter Li, Rick Scheon and Jiaping Wang for their interest in this work. Part of this work was done while the author was visiting the Mathematics Department of Stanford University. The author would like to thank the Department of Mathematics for their hospitality.

\section{REFERENCES}

[G] A. A. Grigor'yan, The heat equation on noncompact Riemannian manifolds (Russian), Mat. Sbornik, vol. 182 (1), 1991, pp. 55-87; Engl. Translation in Math. USSR Sbornik Vol 72 (1) 1992 page 47-77. MR 92h:58189

[K-S] S. Kusuoka and D. Strook, Applications of the Malliavin calculus. III, J. Fac. Sci. Univ. Tokyo, Sect. IA Math. 34 (1987), 391-442. MR 89c:60093

[L] P. Li, Large time behavior of the heat equation on complete manifolds with nonnegative Ricci curvature, Ann. Math. 124 (1986), 1-21. MR 87k:58259

[L-W] P. Li and J. Wang, Mean value inequalities, Indiana Math. J. 48 (1999), 1257-1283. MR 2001e: 58032

[S-T-W] C. J. Sung, L. F. Tam and J. Wang, Spaces of harmonic functions, J. London Math. Soc. 61 (2000), 789-806. MR 2001i:31013 
[Sc] L. Saloff-Coste, A note on Poincaré, Sobolev and Harnack inequalities, Internat. Math. Res. 2 (1992), 27-38. MR 93d:58158

[W] J. Wang, Global heat kernel estimates, Pacific J. Math. 178 (1997), 377-398. MR 98g:58168

Department of Mathematics, National Chung Cheng University, Chia-Yi, Taiwan 62117

E-mail address: cjsung@math.ccu.edu.tw 